\title{
Smoothness of moments of the solutions of discrete coagulation equations with diffusion
}

\author{
Maxime Breden $^{1}$. Laurent Desvillettes ${ }^{2}$. \\ Klemens Fellner ${ }^{3}$ (DD
}

Received: 1 April 2016 / Accepted: 7 September 2016 / Published online: 19 September 2016 (C) The Author(s) 2016. This article is published with open access at Springerlink.com

\begin{abstract}
In this paper, we establish smoothness of moments of the solutions of discrete coagulation-diffusion systems. As key assumptions, we suppose that the coagulation coefficients grow at most sub-linearly and that the diffusion coefficients converge towards a strictly positive limit (those conditions also imply the existence of global weak solutions and the absence of gelation).
\end{abstract}

Keywords Discrete coagulation systems - Smoluchowski equations - Duality arguments $\cdot$ Regularity $\cdot$ Smoothness $\cdot$ Moments estimates

Mathematics Subject Classification 35B45 $\cdot 35 B 65 \cdot 82 \mathrm{D} 60$

Communicated by A. Jüngel.

$凶 \quad$ Klemens Fellner

klemens.fellner@uni-graz.at

Maxime Breden

breden@cmla.ens-cachan.fr

Laurent Desvillettes

desvillettes@math.univ-paris-diderot.fr

1 CMLA, ENS Cachan, CNRS, Université Paris-Saclay, 61 avenue du Président Wilson, 94235 Cachan, France

2 University Paris Diderot, Sorbonne Paris Cité, Institut de Mathématiques de Jussieu-Paris Rive Gauche, UMR 7586, CNRS, Sorbonne Universités, UPMC University Paris 06, Batiment Sophie Germain, 8 Place Aurélie Nemours, 75013 Paris, France

3 Institute of Mathematics and Scientific Computing, NAWI Graz, University of Graz, Heinrichstr. 36, 8010 Graz, Austria 


\section{Introduction}

In this paper we consider discrete coagulation systems with spatial diffusion. Coagulation models appear in a wide range of applications ranging from chemistry (e.g. the formation of polymers) over physics (aerosols, raindrops, smoke, sprays), astronomy (the formation of galaxies) to biology (haematology, animal grouping), see e.g. the surveys $[10,12,20]$ and the references therein.

Following the pioneering works of Smoluchowski (see [26,27]), we shall denote by $c_{i}:=c_{i}(t, x) \in \mathbb{R}_{+}$the concentration of polymers or clusters of mass/size $i \in \mathbb{N}^{*}$ at time $t$ and position $x$. Here, we consider a smooth bounded domain $\Omega$ of $\mathbb{R}^{N}$ in which the clusters are confined via homogeneous Neumann conditions (in applications, we of course have $N \leq 3$ ). Moreover, for any positive time $T$, we denote by $\Omega_{T}$ the set $[0, T] \times \Omega$.

We assume that the concentrations $c_{i}$ satisfy the following infinite (for all $i \in \mathbb{N}^{*}:=$ $\mathbb{N} \backslash\{0\}$ ) set of reaction-diffusion equations with homogeneous Neumann boundary conditions:

$$
\begin{cases}\partial_{t} c_{i}-d_{i} \Delta_{x} c_{i}=Q_{i}(c) & \text { on } \Omega_{T} \\ \nabla_{x} c_{i} \cdot v=0 & \text { on }[0, T] \times \partial \Omega, \\ c_{i}(0, \cdot)=c_{i}^{i n} & \text { on } \Omega,\end{cases}
$$

where $d_{i}>0$ are strictly positive diffusion coefficients, $v(x)$ denotes the outward unit normal vector at point $x \in \partial \Omega$ and $c_{i}^{i n}$ are given initial data, which are typically assumed nonnegative.

The coagulation terms $Q_{i}(c)$ depend on the whole sequence of concentrations $c=\left(c_{i}\right)_{i \in \mathbb{N}^{*}}$ and can be written as the difference between a gain term $Q_{i}^{+}(c)$ and a loss term $Q_{i}^{-}(c)$, which take the form

$$
Q_{i}(c):=Q_{i}^{+}(c)-Q_{i}^{-}(c)=\frac{1}{2} \sum_{j=1}^{i-1} a_{i-j, j} c_{i-j} c_{j}-\sum_{j=1}^{\infty} a_{i, j} c_{i} c_{j}
$$

Here, the nonnegative parameters $a_{i, j}$ represent the coagulation coefficients of clusters of size $i$ merging with clusters of size $j$, which are symmetric: $a_{i, j}=a_{j, i}$. In this work, we consider the case in which the coagulation coefficients additionally satisfy the following asymptotic behaviour:

$$
\lim _{j \rightarrow \infty} \frac{a_{i, j}}{j}=0, \quad \forall i \in \mathbb{N}^{*}, \quad 0 \leq a_{i, j}=a_{j, i}, \quad \forall i, j \in \mathbb{N}^{*}
$$

The conditions (3) are sufficient to provide the existence of global $L^{1}$-weak solutions (with nonnegative concentrations) to system (1), (2) (for which the below estimate (8) on the mass holds), when suitable nonnegative initial data are considered, see [19].

Thanks to the symmetry assumption on the coagulation coefficients, we can write (at a formal level) the following weak formulation of the coagulation operator: for any test-sequence $\left(\varphi_{i}\right)_{i \in \mathbb{N}^{*}}$, we have 


$$
\sum_{i=1}^{\infty} \varphi_{i} Q_{i}(c)=\frac{1}{2} \sum_{i=1}^{\infty} \sum_{j=1}^{\infty} a_{i, j} c_{i} c_{j}\left(\varphi_{i+j}-\varphi_{i}-\varphi_{j}\right)
$$

In the sequel, we shall systematically denote for any $k \in \mathbb{R}_{+}$by

$$
\rho_{k}(t, x):=\sum_{i=1}^{\infty} i^{k} c_{i}(t, x)
$$

the moment of order $k$ of the sequence of concentrations $\left(c_{i}\right)_{i \in \mathbb{N}^{*}}$ and, similarly, the moment of order $k$ of the initial concentrations by

$$
\rho_{k}^{i n}(x):=\sum_{i=1}^{\infty} i^{k} c_{i}^{i n}(x) .
$$

By taking $\varphi_{i}=i$ in (4), we see that (still at a formal level) the conservation of the total mass contained in all clusters/polymers holds, that is,

$\forall t \geq 0, \quad \int_{\Omega} \rho_{1}(t, x) d x=\int_{\Omega} \sum_{i=1}^{\infty} i c_{i}(t, x) d x=\int_{\Omega} \sum_{i=1}^{\infty} i c_{i}^{i n}(x) d x=\int_{\Omega} \rho_{1}^{i n}(x) d x$.

It is a well-known phenomenon for coagulation models, called gelation (see for instance $[12,13])$, that the formal conservation of the total mass (7) will not hold for solutions of coagulation models with sufficiently growing coagulation coefficients $a_{i, j}$ (already for space homogeneous models): When approximating the first order moment

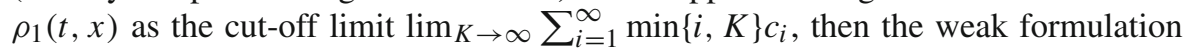
(4) for the test-sequence $\varphi_{i}=\min \{i, K\}$ shows that the map $t \mapsto \sum_{i=1}^{\infty} \min \{i, K\} c_{i}$ is non-increasing in time, and Fatou's lemma only implies that the total mass is nonincreasing in time (for space homogeneous and space inhomogeneous coagulation with homogeneous Neumann boundary conditions models alike). The conservation law (7) can become a strict inequality for solutions of (1) with sufficiently growing coagulation coefficients, but we still get a natural uniform-in-time bound in $L^{\infty}\left(\mathbb{R}_{+} ; L^{1}(\Omega)\right)$ of the total mass $\rho_{1}$, namely

$$
\forall t \geq 0, \quad \int_{\Omega} \rho_{1}(t, x) d x \leq \int_{\Omega} \rho_{1}^{i n}(x) d x .
$$

A standard way to prove the existence of weak solutions to system (1), (2) satisfying (8) is to consider a sequence of truncated systems for which we can prove existence of smooth solutions. Then, using some compactness arguments, one extracts a solution of the limiting system (1), (2), again see for instance [19]. In this work, for any $n \in \mathbb{N}^{*}$, we define $c^{n}=\left(c_{1}^{n}, \ldots, c_{n}^{n}\right)$ as the solution of the truncated problem: $\forall 1 \leq i \leq n$,

$$
\begin{cases}\partial_{t} c_{i}^{n}-d_{i} \Delta_{x} c_{i}^{n}=Q_{i}^{n}\left(c^{n}\right) & \text { on } \Omega_{T} \\ \nabla_{x} c_{i}^{n} \cdot v=0 & \text { on }[0, T] \times \partial \Omega, \\ c_{i}^{n}(0, \cdot)=c_{i}^{i n} & \text { on } \Omega\end{cases}
$$


where

$$
Q_{i}^{n}\left(c^{n}\right)=\frac{1}{2} \sum_{j=1}^{i-1} a_{i-j, j} c_{i-j}^{n} c_{j}^{n}-\sum_{j=1}^{n-i} a_{i, j} c_{i}^{n} c_{j}^{n} .
$$

This is now a finite system of reaction-diffusion equations with finite sums in the r.h.s, for which the existence and uniqueness of nonnegative, global and smooth solutions have already been proven (see for example Proposition 2.1 and Lemma 2.2 of [29], or [9]). Notice that for any sequence $\left(\varphi_{i}\right)_{i \in \mathbb{N}^{*}}$, we have

$$
\sum_{i=1}^{n} \varphi_{i} Q_{i}^{n}\left(c^{n}\right)=\frac{1}{2} \sum_{i+j \leq n ; i, j \geq 1} a_{i, j} c_{i}^{n} c_{j}^{n}\left(\varphi_{i+j}-\varphi_{i}-\varphi_{j}\right)
$$

so that we get (this time rigorously)

$$
\forall t \geq 0, \quad \int_{\Omega} \sum_{i=1}^{n} i c_{i}^{n}(t, x) d x=\int_{\Omega} \sum_{i=1}^{n} i c_{i}^{i n}(x) d x .
$$

If we manage to extract a limit from $\left(c_{n}\right)$, Fatou's Lemma then yields (8) for the limiting concentration.

Before proceeding further, let us introduce a precise definition of weak solution, following [19].

Definition 1.1 A global weak solution $c=\left(c_{i}\right)_{i \in \mathbb{N}^{*}}$ to (1), (2) is a sequence of functions $c_{i}:[0,+\infty) \times \Omega \rightarrow[0,+\infty)$ such that, for all $i \in \mathbb{N}^{*}$ and $T>0$

- $c_{i} \in \mathcal{C}\left([0, T] ; L^{1}(\Omega)\right)$,

- $Q_{i}^{-}(c) \in L^{1}\left(\Omega_{T}\right)$,

- $\sup _{t \geq 0} \int_{\Omega} \rho_{1}(t, x) d x \leq \int_{\Omega} \rho_{1}^{i n}(x) d x$,

- $c_{i}$ is a mild solution to the $i$-th equation in (1), that is

$$
c_{i}(t)=e^{d_{i} A_{1} t} c_{i}^{i n}+\int_{0}^{t} e^{d_{i} A_{1}(t-s)} Q_{i}(c(s)) d s,
$$

where $Q_{i}$ is defined by (2), $A_{1}$ is the closure in $L^{1}(\Omega)$ of the unbounded, linear, self-adjoint operator $A$ of $L^{2}(\Omega)$ defined by

$$
D(A)=\left\{w \in H^{2}(\Omega), \nabla w \cdot v=0 \text { on } \partial \Omega\right\}, \quad A w=\Delta w,
$$

and $e^{d_{i} A_{1} t}$ is the $\mathcal{C}^{0}$-semigroup generated by $d_{i} A_{1}$ in $L^{1}(\Omega)$.

The following result, which is a direct application of [19, Theorem 3], states that we can obtain weak solution of (1), (2) from the truncated systems (9), (10). We also refer to $[29,30]$. 
Proposition 1.2 Let $\Omega$ be a smooth bounded domain of $\mathbb{R}^{N}$. Assume that the coagulation coefficients satisfy (3) and that all diffusion coefficients are strictly positive, i.e. $d_{i}>0 \forall i \in \mathbb{N}^{*}$. Assume also that the initial concentrations $c_{i}^{i n} \geq 0$ are such that $\rho_{1}^{i n} \in L^{1}(\Omega)$. For every $n \in \mathbb{N}^{*}$, let $c^{n}=\left(c_{1}^{n}, \ldots, c_{n}^{n}\right)$ be the solution of the truncated system of size $n$ (9), (10).

Then, there exists a sequence $c=\left(c_{i}\right)_{i \in \mathbb{N}^{*}}$ such that, up to extraction

$$
c_{i}^{n} \underset{n \rightarrow \infty}{\longrightarrow} c_{i} \text { in } L^{1}\left(\Omega_{T}\right), \quad \forall i \in \mathbb{N}^{*}, \forall T>0
$$

and c is a weak solution to (1), (2) in the sense of Definition 1.1.

Our first proposition states that if the diffusion rates of clusters of different sizes are sufficiently close to each others, the natural uniform $L^{1}$-bound (8) can be extended to $L^{p}$ (with $p>1$ depending on the closeness of the diffusion rates). To be more precise about this closeness hypothesis, let us first introduce

Definition 1.3 For $m>0$ and $q \in] 1,+\infty$ [, we define $\mathcal{K}_{m, q}>0$ as the best (i.e. the smallest) constant independent of $T>0$ in the parabolic regularity estimate

$$
\left(\int_{\Omega_{T}}\left|\partial_{t} v\right|^{q}+m^{q} \int_{\Omega_{T}}\left|\Delta_{x} v\right|^{q}\right)^{\frac{1}{q}} \leq \mathcal{K}_{m, q}\left(\int_{\Omega_{T}}|f|^{q}\right)^{\frac{1}{q}}, \quad \forall f \in L^{q}\left(\Omega_{T}\right),
$$

where $v$ is the unique solution of the heat equation with constant diffusion coefficient $m$, homogeneous Neumann boundary conditions and zero initial data:

$$
\begin{cases}\partial_{t} v-m \Delta_{x} v=f & \text { on } \Omega_{T} \\ \nabla_{x} v \cdot v=0 & \text { on }[0, T] \times \partial \Omega \\ v(0, \cdot)=0 & \text { on } \Omega\end{cases}
$$

The existence of such a constant $\mathcal{K}_{m, q}<\infty$ independent of the time $T>0$ is explicitly stated in [18] provided that $\partial \Omega \in \mathcal{C}^{2+\alpha}, \alpha>0$.

Next, we present the

Proposition 1.4 Let $\Omega$ be a smooth bounded domain of $\mathbb{R}^{N}$ (e.g. $\partial \Omega \in \mathcal{C}^{2+\alpha}, \alpha>0$ ). Let $p \in] 1,+\infty\left[\right.$ and assume that the nonnegative initial data $c_{i}^{i n} \geq 0$ have an initial mass $\rho_{1}^{i n}$ which lies in $L^{p}(\Omega)$. Assume that the coagulation coefficients satisfy (3). Assume that

$$
0<\delta:=\inf _{i \geq 1} d_{i}, \quad \text { and } \quad D:=\sup _{i \geq 1} d_{i}<\infty .
$$

Then, provided that for the Hölder conjugate $p^{\prime}$ of $p$ holds the condition

$$
\frac{D-\delta}{D+\delta} \mathcal{K}_{\frac{D+\delta}{2}, p^{\prime}}<1
$$

there exists a weak solution of the coagulation system (1), (2) such that the mass $\rho_{1}$ lies in $L^{p}\left(\Omega_{T}\right)$ for any finite time $T>0$. 
Remark 1.5 Note that the above estimate was already proven in [5] in the particular case $p=2$, even without assuming (13). In fact, the Hilbert space case $p=2$ allows to prove the explicit bound $\mathcal{K}_{m, 2} \leq 1$ (see Lemma 4.4), which leads to

$$
\frac{D-\delta}{D+\delta} \mathcal{K}_{\frac{D+\delta}{2}, 2} \leq \frac{D-\delta}{D+\delta}<1
$$

and hypothesis (13) is automatically satisfied for $p=2$ for all $0<\delta \leq D<\infty$ and $T>0$ (hence its absence in [5]).

Note that this global $L^{2}$-bound together with assumptions (3) also ensures that no gelation can occur, so that the conservation law (7) rigorously holds for any weak solution, see [5].

Moreover, the strict inequality in (14) has been further exploited in [6] by proving a continuous upper bound of the best constant $\mathcal{K}_{m, p^{\prime}}$ on $p^{\prime} \leq 2$. Therefore, for all $0<\delta \leq D<\infty$, there exists a sufficiently small $0<\varepsilon=\varepsilon(\delta, D) \ll 1$ such that (14) can be slightly improved to

$$
\frac{D-\delta}{D+\delta} \mathcal{K}_{\frac{D+\delta}{2}, 2-O(\varepsilon)}<1,
$$

and this allows to prove a correspondingly improved a priori estimate in $L^{2+\varepsilon}\left(\Omega_{T}\right)$.

Proposition 1.4 can be improved in the case when the diffusion coefficients $\left(d_{i}\right)_{i \in \mathbb{N}^{*}}$ constitute a sequence converging towards a strictly positive limit. Note that such an assumption is not so far from the assumption that the sequence $\left(d_{i}\right)_{i \in \mathbb{N}^{*}}$ is bounded above and below (by a strictly positive constant), which is used in Proposition 1.4 (or also in [5]), since one expects on physical grounds that the sequence $\left(d_{i}\right)_{i \in \mathbb{N}^{*}}$ is decreasing; that is, that larger clusters diffuse less. Under this assumption and provided that the coagulation coefficients are strictly sublinear (see the precise assumption in Theorem 1.6 below) we can show that $L^{p}$ norms of moments $\rho_{k}$ are propagated for any $\left.k \in \mathbb{N}^{*}, p \in\right] 1, \infty[$.

Theorem 1.6 Let $\Omega$ be a smooth bounded domain of $\mathbb{R}^{N}$. Assume that the coagulation coefficients satisfy for a constant $C>0$ and all $i, j \in \mathbb{N}^{*}$

$$
0 \leq a_{i, j}=a_{j, i} \leq C\left(i^{\gamma}+j^{\gamma}\right), \text { for some } \gamma \in[0,1[\text {, }
$$

and that $\left(d_{i}\right)_{i \in \mathbb{N}}$ is a sequence of strictly positive real numbers which converges toward a strictly positive limit.

Assume that (for some $k \in \mathbb{N}^{*}$ ) the initial moment $\rho_{k}^{\text {in }}$ lies in $L^{p}(\Omega)$ for all $p<+\infty$ and that (for all $i \in \mathbb{N}^{*}$ ) each initial concentration $c_{i}^{i n} \geq 0$ lies in $L^{\infty}(\Omega)$.

Then, there exists a global weak nonnegative solution to (1), (2) for which the moment $\rho_{k}$ lie in $L^{p}\left(\Omega_{T}\right)$ for all $p<+\infty$ and all finite time $T>0$.

Remark 1.7 Notice that hypothesis (15) on the coagulation coefficients implies the assumption (3), which in return yields existence of global weak solutions. 
In the existing literature, sublinear assumptions on the coagulation coefficients are often found under the form:

$$
0 \leq a_{i, j}=a_{j, i} \leq \tilde{C}\left(i^{\alpha} j^{\beta}+i^{\beta} j^{\alpha}\right), \quad \text { for some } \alpha, \beta \in[0,1[\text { with } \alpha+\beta<1 \text {. }
$$

Our motive for using assumption (15) rather than assumption (16) is mainly that it allows for slightly shorter computations, without any loss of generality since (16) implies (15) with $\gamma=\alpha+\beta$.

Remark 1.8 We point out that Theorem 1.6 could be extended to the case where a finite number of diffusion coefficients $d_{i}$ are equal to 0 (see Remark 3.7).

In the case that $d_{i}=0$ starting from some $i=I$ as considered for instance in [31], our approach should allow for a corresponding generalisation of Theorem 1.6 provided that a suitable "closeness" condition on the finitely many non-zero diffusion coefficients is satisfied.

The study of moments for the coagulation equation has been a longstanding strategy to get mass conservation and uniqueness results (see [22] for one of the first work in this direction for the coagulation equation with diffusion, in the continuous case).

More recently, results in the same spirit as Proposition 1.6 about propagation of moments have been obtained in [24,25], where the system (1), (2) and its continuous counterpart are studied on the whole space $\mathbb{R}^{N}$. Assuming (12) and a finite total increase of variation for $\left(d_{i}\right)$, together with a control on the growth of the coagulation coefficients (also involving the diffusion rates) such as

$$
\underset{(i+j)\left(d_{i}+d_{j}\right)}{\underset{i+j \rightarrow+\infty}{\longrightarrow}} 0,
$$

and the finiteness of some of the initial moments (in different norms), bounds are obtained which look like

$$
\left\|\rho_{k}(t, \cdot)\right\|_{L^{p}(\Omega)} \leq\left\|\rho_{k}^{i n}\right\|_{L^{p}(\Omega)}+C k^{-l}
$$

where $l$ depends on the degree of the initial moments assumed to be finite.

The statement of our result is therefore close to that of [25] (our requirement on the diffusion rate is however more stringent), but the proof is completly different, so that the exact conditions required on initial data are also different. Note that the limit case $a_{i, j}=i+j$ is still open (absence of gelation for this coagulation coefficient is conjectured in general, but is proven only when there is no diffusion, see for instance $[4,28])$.

Although in the present work, $L^{p}$ estimates for moments are only shown for $p<\infty$ (whereas $p=\infty$ can be obtained in [25]), the use of parabolic inequalities for the heat equation enables to recover this case (and also higher order derivatives).

Indeed, the estimates obtained in Theorem 1.6 can be improved if the initial data are assumed to be smooth enough. This leads to our main Theorem, namely: 
Theorem 1.9 Let $\Omega$ be a smooth bounded domain of $\mathbb{R}^{N}$. Assume that the coagulation coefficients satisfy (15) and that $\left(d_{i}\right)_{i \in \mathbb{N}}$ is a sequence of strictly positive real numbers which converges toward a strictly positive limit.

Assume that the initial data $c_{i}^{i n} \geq 0$ are of class $\mathcal{C}^{\infty}(\bar{\Omega})$, compatible with the boundary conditions, and that for all $k \in \mathbb{N}^{*}$ the initial moments $\rho_{k}^{\text {in }}$ are of class $\mathcal{C}^{\infty}(\bar{\Omega})$.

Then, there exist a unique smooth solution to (1), (2) such that each $\left(c_{i}\right)$ is nonnegative, of class $\mathcal{C}^{\infty}\left(\bar{\Omega}_{T}\right)$ for any finite time $T>0$, and such that the moments $\rho_{k}$ are also of class $\mathcal{C}^{\infty}\left(\bar{\Omega}_{T}\right)$, for any $k \in \mathbb{N}^{*}$.

Remark 1.10 The $\mathcal{C}^{\infty}$ regularity down to time 0 requires of course the $\mathcal{C}^{\infty}$ hypothesis on the initial data. However, it can be seen in the various steps of the proof (see Sect. 4) that propagation of regularity in intermediate Sobolev spaces holds under suitable (less stringent) assumptions on the initial data.

Since each $c_{i}$ is solution of a heat equation subject to a r.h.s. that can be controlled once all moments are bounded in $L^{p}\left(\Omega_{T}\right), p<+\infty$, we can in fact show the creation of regularity for strictly positive times. For example, under the assumption that $\rho_{k}^{i n} \in$ $L^{p}(\Omega)$ for all $p<+\infty$ and all $k \in \mathbb{N}^{*}$, we can prove that the concentrations $c_{i}$ are of class $\left.\left.C^{\infty}(] 0, T\right] \times \bar{\Omega}\right)$.

Also, as will be made clear in Sect. $4, \mathcal{C}^{\infty}$ regularity is not needed to ensure uniqueness. As shown in [15], uniqueness holds as soon as $\rho_{2} \in L^{\infty}$, so that starting from initial data leading to an estimate for $\rho_{2}$ in a Sobolev space embedded in $L^{\infty}$, uniqueness can already be obtained.

Finally, we point out that assumption (15) is not far from optimal, since it is known that gelation can occur as soon as $a_{i, j}=i^{\alpha} j^{\beta}+i^{\beta} j^{\alpha}$ with $\alpha+\beta>1$ (see [13]) and gelation is not compatible with the conclusion of Theorems 1.6 or 1.9.

Our paper is organized as follows. In Sect. 2, we recall some lemmas existing in the literature and called duality lemmas. We also introduce modified versions of those lemmas, that are later used in Sect. 3 to prove the propagation of moments in $L^{p}\left(\Omega_{T}\right)$, $p<+\infty$ (Propositions 1.4 and 1.6). In Sect. 4, we extend these results to prove $\mathcal{C}^{\infty}$ regularity for the concentrations and the moments (Theorem 1.9). Finally, a short Appendix is devoted to technical lemmas which are useful to make the proof of some duality lemmas rigorous.

\section{Duality estimates}

We start by recalling some a priori estimates based on duality arguments from [6]. These estimates are key ingredients of the present work. In this section, functions said to be weak solutions ought to be understood as solutions of the equation obtained by multiplying by a test function and integrating by parts. Remember also that $\mathcal{K}_{m, q}$ is defined in Definition 1.3.

The first statement recalls [6, Lemma 2.2].

Lemma 2.1 Let $\Omega$ be a smooth bounded subset of $\mathbb{R}^{N}$ and consider a function $M:=$ $M(t, x): \Omega_{T} \rightarrow \mathbb{R}_{+}$satisfying $a \leq M \leq b$ for some $a, b>0$. For any $\left.q \in\right] 1,+\infty[$, if 


$$
\frac{b-a}{b+a} \mathcal{K}_{\frac{a+b}{2}, q}<1
$$

then, there exist constants $C_{0}>0$ and $C>0$ (depending on $\Omega, a, b, q, T$ ) such that for any $f \in L^{q}(\Omega)$, the (unique, weak) solution $v$ of the backward parabolic system with $L^{\infty}$ coefficient $M:=M(t, x)$,

$$
\begin{cases}\partial_{t} v+M \Delta_{x} v=f & \text { on } \Omega_{T}, \\ \nabla_{x} v \cdot v=0 & \text { on }[0, T] \times \partial \Omega, \\ v(T, \cdot)=0 & \text { on } \Omega,\end{cases}
$$

satisfies $\|v\|_{L^{q}\left(\Omega_{T}\right)} \leq C\|f\|_{L^{q}\left(\Omega_{T}\right)}$ and $\|v(0, \cdot)\|_{L^{q}(\Omega)} \leq C_{0}\|f\|_{L^{q}\left(\Omega_{T}\right)}$.

Remark 2.2 The bound on $\|v\|_{L^{q}\left(\Omega_{T}\right)}$ is not explicitly mentioned in Lemma 2.2 of [6], but is a direct consequence of its proof, in particular of the estimates $\left\|\Delta_{x} v\right\|_{L^{q}\left(\Omega_{T}\right)} \leq$ $C_{1}\|f\|_{L^{q}\left(\Omega_{T}\right)}$ and $\left\|\partial_{t} v\right\|_{L^{q}\left(\Omega_{T}\right)} \leq C_{1}\|f\|_{L^{q}\left(\Omega_{T}\right)}$, which are explicitly mentioned there.

Remark 2.3 The fact that the above mentioned function $v$ exists (and is unique) is (in particular for $q<2$ ) not obvious because $M$ is not assumed to be continuous (or at least VMO). In the Appendix (Proposition 4.3), we give a proof of the existence and uniqueness of $v$ for the sake of completeness.

Lemma 2.1 is used to prove the following duality lemma, which is Proposition 1.1 of [6].

Proposition 2.4 Let $\Omega$ be a smooth bounded subset of $\mathbb{R}^{N}$ and consider a function $M:=M(t, x): \Omega_{T} \rightarrow \mathbb{R}_{+}$satisfying $a \leq M \leq b$ for some $a, b>0$. For any $p \in] 1,+\infty[$, if

$$
\frac{b-a}{b+a} \mathcal{K}_{\frac{a+b}{2}, p^{\prime}}<1,
$$

then, there exists a constant $C>0$ (depending on $\Omega, a, b, p, T$ ) such that for any $u_{0} \in L^{p}(\Omega)$, any weak solution $u$ of the parabolic system (in divergence form)

$$
\begin{cases}\partial_{t} u-\Delta_{x}(M u)=0 & \text { on } \Omega_{T}, \\ \nabla_{x} u \cdot v=0 & \text { on }[0, T] \times \partial \Omega, \\ u(0, \cdot)=u_{0} & \text { on } \Omega,\end{cases}
$$

satisfies $\|u\|_{L^{p}\left(\Omega_{T}\right)} \leq C\left\|u_{0}\right\|_{L^{p}(\Omega)}$.

In the sequel we will need a generalized version of Proposition 2.4, which is an adaptation of Theorem 3.1 in [11] (where only the case $p=2$ is treated).

Proposition 2.5 Let $\Omega$ be a smooth bounded subset of $\mathbb{R}^{N}, \mu_{1}, \mu_{2} \geq 0$, and consider a function $M:=M(t, x): \Omega_{T} \rightarrow \mathbb{R}_{+}$satisfying $a \leq M \leq b$ for some $a, b>0$. For any $p \in] 1,+\infty[$, if

$$
\frac{b-a}{b+a} \mathcal{K}_{\frac{a+b}{2}, p^{\prime}}<1,
$$


then, there exists a constant $C>0$ (depending on $\Omega, a, b, p, \mu_{1}, \mu_{2}, T$ ) such that for any $u_{0} \in L^{p}(\Omega)$, any function $u: \Omega_{T} \rightarrow \mathbb{R}_{+}$satisfying (weakly)

$$
\begin{cases}\partial_{t} u-\Delta_{x}(M u) \leq \mu_{1} u+\mu_{2} & \text { on } \Omega_{T} \\ \nabla_{x} u \cdot v=0 & \text { on }[0, T] \times \partial \Omega \\ u(0, \cdot)=u_{0} & \text { on } \Omega\end{cases}
$$

belongs to $L^{p}\left(\Omega_{T}\right)$, with the estimate:

$$
\|u\|_{L^{p}\left(\Omega_{T}\right)} \leq C\left(1+\left\|u_{0}\right\|_{L^{p}(\Omega)}\right) .
$$

Proof Let $\varphi$ be a nonnegative smooth function on $\Omega_{T}$ and $v$ be the (unique, weak) solution (cf. Proposition 4.3) of the dual problem

$$
\begin{cases}\partial_{t} v+M \Delta_{x} v+\mu_{1} v=-\varphi & \text { on } \Omega_{T} \\ \nabla_{x} v \cdot v=0 & \text { on }[0, T] \times \partial \Omega \\ v(T, \cdot)=0 & \text { on } \Omega\end{cases}
$$

Notice that the function $\tilde{v}$ defined by $\tilde{v}(t, x)=v(T-t, x)$ satisfies a standard, forward in time, reaction-diffusion equation

$$
\begin{cases}\partial_{t} \tilde{v}-\tilde{M} \Delta_{x} \tilde{v}=\mu_{1} \tilde{v}+\tilde{\varphi} & \text { on } \Omega_{T} \\ \nabla_{x} \tilde{v} \cdot v=0 & \text { on }[0, T] \times \partial \Omega \\ \tilde{v}(0, \cdot)=0 & \text { on } \Omega\end{cases}
$$

where $\tilde{M}(t, x)=M(T-t, x)$ and $\tilde{\varphi}(t, x)=\varphi(T-t, x)$. This ensures that $\tilde{v}$, and therefore $v$, are nonnegative. Multiplying (19) by the solution $v$ of the dual problem and integrating on $\Omega_{T}$, we end up with

$$
\int_{\Omega_{T}} u \varphi \leq \int_{\Omega} u_{0} v(0)+\mu_{2} \int_{\Omega_{T}} v \leq\left\|u_{0}\right\|_{L^{p}(\Omega)}\|v(0)\|_{L^{p^{\prime}}(\Omega)}+\mu_{2}(|\Omega| T)^{\frac{1}{p}}\|v\|_{L^{p^{\prime}}\left(\Omega_{T}\right)} .
$$

Moreover, the rescaled function $w=e^{\mu_{1} t} v$ satisfies

$$
\begin{cases}\partial_{t} w+M \Delta_{x} w=-e^{\mu_{1} t} \varphi & \text { on } \Omega_{T} \\ \nabla_{x} w \cdot v=0 & \text { on }[0, T] \times \partial \Omega \\ w(T, \cdot)=0 & \text { on } \Omega\end{cases}
$$

Thus, provided that hypothesis (18) is satisfied, we can apply Lemma 2.1 to $w$ and get (after noticing that $|v| \leq|w|$ )

$$
\|v\|_{L^{p^{\prime}}\left(\Omega_{T}\right)} \leq C\|\varphi\|_{L^{p^{\prime}}\left(\Omega_{T}\right)}, \quad \text { and } \quad\|v(0)\|_{L^{p^{\prime}}(\Omega)} \leq C_{0}\|\varphi\|_{L^{p^{\prime}}\left(\Omega_{T}\right)}
$$


where the term $e^{\mu_{1} T}$ is absorbed in the constants. Returning to (20), we finally obtain

$$
\int_{\Omega_{T}} u \varphi \leq C\left(1+\left\|u_{0}\right\|_{L^{p}(\Omega)}\right)\|\varphi\|_{L^{p^{\prime}\left(\Omega_{T}\right)}},
$$

for all nonnegative smooth functions $\varphi$, and the statement of Proposition 2.5 follows by duality.

We finish this section with another variant of the duality lemma, in which $L^{p}$ r.h.s. can be treated.

Proposition 2.6 Let $\Omega$ be a smooth bounded subset of $\mathbb{R}^{N}$ and consider a function $M:=M(t, x): \Omega_{T} \rightarrow \mathbb{R}_{+}$satisfying $a \leq M \leq b$ for some $a, b>0$. Consider functions $A$ and $B$ defined on $\Omega_{T}$ and a real number $\left.\varepsilon \in\right] 0,1[$. Assume that for some $p \in] 1,+\infty[$, the following statements hold:

$$
\frac{b-a}{b+a} \mathcal{K}_{\frac{a+b}{2}, p^{\prime}}<1, \quad A \in L^{\frac{p}{\varepsilon}}\left(\Omega_{T}\right), \quad B \in L^{p}\left(\Omega_{T}\right) .
$$

Then, there exists a constant $C$ (depending on $\Omega, a, b, p, \varepsilon, T$ ) such that for any $u_{0} \in L^{p}(\Omega)$, and any nonnegative $u \in L^{p}\left(\Omega_{T}\right)$ satisfying (weakly)

$$
\begin{cases}\partial_{t} u-\Delta_{x}(M u) \leq A u^{1-\varepsilon}+B & \text { on } \Omega_{T}, \\ \nabla_{x} u \cdot v=0 & \text { on }[0, T] \times \partial \Omega, \\ u(0, \cdot)=u_{0} & \text { on } \Omega,\end{cases}
$$

the following estimate holds:

$$
\|u\|_{L^{p}\left(\Omega_{T}\right)}^{p} \leq C\left(\left\|u_{0}\right\|_{L^{p}(\Omega)}^{p}+\|A\|_{L^{\frac{p}{\varepsilon}\left(\Omega_{T}\right)}}^{\frac{p}{\varepsilon}}+\|B\|_{L^{p}\left(\Omega_{T}\right)}^{p}\right) .
$$

Remark 2.7 We stress the fact that Proposition 2.6 above requires a priori that the function $u$ lies in $L^{p}\left(\Omega_{T}\right)$. As a consequence, we shall not be able to directly apply this result to weak solutions of (1), (2), but only to solutions of an approximate (truncated) system (such as (9), (10)), for which we have a priori regularity estimates.

Proof We consider $v$ the solution (whose existence and uniqueness are once again given by Proposition 4.3 of the Appendix) of the dual problem

$$
\begin{cases}\partial_{t} v+M \Delta_{x} v=-u^{p-1} & \text { on } \Omega_{T}, \\ \nabla_{x} v \cdot v=0 & \text { on }[0, T] \times \partial \Omega, \\ v(T, \cdot)=0 & \text { on } \Omega .\end{cases}
$$

Again multiplying (22) by $v$ and integrating on $\Omega_{T}$, we end up with

$$
\int_{\Omega_{T}} u^{p} \leq \int_{\Omega} u_{0} v(0)+\int_{\Omega_{T}} A u^{1-\varepsilon} v+\int_{\Omega_{T}} B v .
$$


Moreover thanks to (21), we can apply Lemma 2.1 to the above dual problem and get that

$$
\|v\|_{L^{p^{\prime}\left(\Omega_{T}\right)}} \leq C\left\|u^{p-1}\right\|_{L^{p^{\prime}\left(\Omega_{T}\right)}} \leq C\|u\|_{L^{p}\left(\Omega_{T}\right)}^{p-1} \quad \text { and } \quad\|v(0)\|_{L^{p^{\prime}}(\Omega)} \leq C_{0}\|u\|_{L^{p}\left(\Omega_{T}\right)}^{p-1} .
$$

Next, returning to (23), we can bound each term of the r.h.s. using several times Young's inequality (sometimes using a parameter $\eta>0$ ): For the first term, we get

$$
\int_{\Omega} u_{0} v(0) \leq \frac{1}{p \eta^{p}} \int_{\Omega} u_{0}^{p}+\frac{\eta^{p^{\prime}}}{p^{\prime}} \int_{\Omega} v(0)^{p^{\prime}} \leq \frac{1}{p \eta^{p}} \int_{\Omega} u_{0}^{p}+\frac{C_{0}^{p^{\prime}} \eta^{p^{\prime}}}{p^{\prime}} \int_{\Omega_{T}} u^{p},
$$

while for the second one,

$$
\begin{aligned}
\int_{\Omega_{T}} A u^{1-\varepsilon} v & \leq \frac{1-\varepsilon}{p} \int_{\Omega_{T}} u^{p}+\frac{\eta^{p^{\prime}}}{p^{\prime}} \int_{\Omega_{T}} v^{p^{\prime}}+\frac{\varepsilon}{p \eta^{\frac{p}{\varepsilon}}} \int_{\Omega_{T}} A^{\frac{p}{\varepsilon}} \\
& \leq\left(\frac{1-\varepsilon}{p}+\frac{C^{p^{\prime}} \eta^{p^{\prime}}}{p^{\prime}}\right) \int_{\Omega_{T}} u^{p}+\frac{\varepsilon}{p \eta^{\frac{p}{\varepsilon}}} \int_{\Omega_{T}} A^{\frac{p}{\varepsilon}},
\end{aligned}
$$

and finally for the last one,

$$
\int_{\Omega_{T}} B v \leq \frac{1}{p \eta^{p}} \int_{\Omega_{T}} B^{p}+\frac{\eta^{p^{\prime}}}{p^{\prime}} \int_{\Omega_{T}} v^{p^{\prime}} \leq \frac{1}{p \eta^{p}} \int_{\Omega_{T}} B^{p}+\frac{C^{p^{\prime}} \eta^{p^{\prime}}}{p^{\prime}} \int_{\Omega_{T}} u^{p} .
$$

Putting everything together, we end up with

$$
\int_{\Omega_{T}} u^{p} \leq\left(\frac{1-\varepsilon}{p}+\frac{\left(2 C^{p^{\prime}}+C_{0}^{p^{\prime}}\right) \eta^{p^{\prime}}}{p^{\prime}}\right) \int_{\Omega_{T}} u^{p}+\frac{1}{p \eta^{p}} \int_{\Omega} u_{0}^{p}+\frac{\varepsilon}{p \eta^{\frac{p}{\varepsilon}}} \int_{\Omega_{T}} A^{\frac{p}{\varepsilon}}+\frac{1}{p \eta^{p}} \int_{\Omega_{T}} B^{p},
$$

and by taking $\eta>0$ small enough, we get the announced estimate.

\section{Propagation of moments in $L^{p}$ norms}

This Section is devoted to the proof of propagation in $L^{p}\left(\Omega_{T}\right)(p<+\infty)$ of moments $\rho_{k}$. We begin with Proposition 1.4 and the propagation of the total mass $\rho_{1}$, when the closeness hypothesis (13) on the diffusion coefficients is satisfied.

Proof of Proposition 1.4. For $n \in \mathbb{N}^{*}$, we consider the solution $c^{n}=\left(c_{1}^{n}, \ldots, c_{n}^{n}\right)$ of (9), (10), for which the existence and uniqueness of nonnegative, global and smooth solutions are classical (see for example Proposition 2.1 and Lemma 2.2 of [29], or [9]). Summing up the equations (9) for each $i$, we get 


$$
\partial_{t}\left(\sum_{i=1}^{n} i c_{i}^{n}\right)-\Delta_{x}\left(\sum_{i=1}^{n} i d_{i} c_{i}^{n}\right)=0
$$

which rewrites, when

$$
\rho_{1}^{n}=\sum_{i=1}^{n} i c_{i}^{n} \quad \text { and } \quad M_{1}^{n}:=\frac{\sum_{i=1}^{n} i d_{i} c_{i}^{n}}{\sum_{i=1}^{\infty} i c_{i}^{n}}
$$

as

$$
\partial_{t} \rho_{1}^{n}-\Delta_{x}\left(M_{1}^{n} \rho_{1}^{n}\right)=0 .
$$

Using

$$
a=\inf _{i \geq 1}\left\{d_{i}\right\} \text { and } b=\sup _{i \geq 1}\left\{d_{i}\right\}
$$

we get $a \leq M_{1}^{n} \leq b$ independently of $n$. Proposition 2.4 then yields

$$
\left\|\rho_{1}^{n}\right\|_{L^{p}\left(\Omega_{T}\right)} \leq C\left\|\rho_{1}^{n}(0, \cdot)\right\|_{L^{p}(\Omega)} \leq C\left\|\rho_{1}^{i n}\right\|_{L^{p}(\Omega)},
$$

where $C$ does not depend on $n$. By Proposition 1.2 (or respectively [19, Theorem 3]), we get a weak solution $c=\left(c_{i}\right)_{i \in \mathbb{N}_{*}}$ of (1), (2) defined by (up to extraction)

$$
c_{i}=\lim _{n \rightarrow \infty} c_{i}^{n}
$$

and thanks to Fatou's Lemma, we see that $\left\|\rho_{1}\right\|_{L^{p}\left(\Omega_{T}\right)} \leq C\left\|\rho_{1}^{0}\right\|_{L^{p}(\Omega)}$.

Remark 3.1 In fact Proposition 1.4 would be valid for any weak solution to (1), (2) such that $\sum_{i=1}^{\infty} i Q_{i}(c) \in L^{1}\left(\Omega_{T}\right)$. Indeed, one can then prove that

$$
\partial_{t}\left(\sum_{i=1}^{\infty} i c_{i}\right)-\Delta_{x}\left(\sum_{i=1}^{\infty} i d_{i} c_{i}\right)=0
$$

holds weakly, and one can then directly apply Proposition 2.4 to (24).

The proof of Theorem 1.6 is a bit more involved but still based on the same idea. The outline of the proof is the following: First, we get $L^{\infty}\left(\Omega_{T}\right)$ bounds for each concentration $c_{i}$ and for any finite time $T$. Thus, it is sufficient to prove propagation in $L^{p}$ spaces for tail moments, in which we only consider concentrations $c_{i}$ for $i$ larger than some index $I$. Because we assumed that the $d_{i}$ converge (when $i \rightarrow \infty$ ) towards a strictly positive real number, the closeness hypothesis (13) will always be satisfied for the coefficients $\left(d_{i}\right)_{i \geq I}$ when $I$ is large enough. This allows us to use a similar argument as in Proposition 1.4 to prove the propagation in $L^{p}\left(\Omega_{T}\right)$ of the mass and then of all higher order moments. 
Proof of Theorem 1.6. As for Proposition 1.4, the rigorous way to prove Theorem 1.6 is to get all the needed estimates on the solutions of the truncated problems (9), (10) and then pass to the limit (when $n \rightarrow \infty$ ). However for a clearer exposition of the different arguments, we first derive (sometimes formally) estimates on the whole system (1), (2) and then explain how to pass to the limit in the corresponding estimates on the truncated system. We begin with the following result (which was already noticed in [29]).

Lemma 3.2 Let $\Omega$ be a smooth bounded domain of $\mathbb{R}^{N}$. Assume that the coagulation coefficients satisfy (3) and that $d_{i}>0$ for all $i \in \mathbb{N}^{*}$. Assume also that each $c_{i}^{\text {in }} \geq 0$ lies in $L^{\infty}(\Omega)$. We consider a global weak nonnegative solution of (1), (2) (nonnegative meaning here that $c_{i} \geq 0$ for all $\left.i \in \mathbb{N}^{*}\right)$.

Then, the concentration $c_{i}$ lies in $L^{\infty}\left(\Omega_{T}\right)$ for each integer $i \in \mathbb{N}^{*}$ and any positive time $T>0$.

Proof Since

$$
\partial_{t} c_{1}-d_{1} \Delta_{x} c_{1} \leq Q_{1}^{+}(c)=0,
$$

the maximum principle for the heat equation yields that $c_{1} \in L^{\infty}\left(\Omega_{T}\right)$. Then, we observe that for all $i \geq 2$,

$$
\partial_{t} c_{i}-d_{i} \Delta_{x} c_{i} \leq Q_{i}^{+}(c)
$$

Since the coagulation gain term $Q_{i}^{+}(c)=\frac{1}{2} \sum_{j=1}^{i-1} a_{i, j} c_{i-j} c_{j}$ involves only $c_{j}$ for $j<i$, we can conclude the statement of the lemma by induction.

The proof of Lemma 3.2 shows sufficient conditions under which each $c_{i}$ is bounded on $\Omega_{T}$, but explicit bounds computed in this way would grow very fast with $i$. Thus, there is little hope of obtaining a result on $\rho_{1}$ by directly using this method. However, the knowledge that any finite truncation of $\rho_{1}$ lies in $L^{\infty}\left(\Omega_{T}\right)$ enables us to prove another result of propagation of $L^{p}$ norms for the mass $\rho_{1}$, where the assumption (13) is removed and replaced by the assumption of convergence of the diffusion coefficients $d_{i}$ towards a strictly positive limit.

Lemma 3.3 Let $\Omega$ be a smooth bounded domain of $\mathbb{R}^{N}$. Assume that the coagulation coefficients satisfy (15). Assume also that all $d_{i}$ are strictly positive, and that $\left(d_{i}\right)$ converges toward a strictly positive limit. Finally, assume that each $c_{i}^{i n} \geq 0$ lies in $L^{\infty}(\Omega)$ and that $\rho_{1}^{i n} \in L^{p}(\Omega)$ for some $\left.p \in\right] 1,+\infty[$. We consider a global weak nonnegative solution of (1), (2) (nonnegative meaning here that $c_{i} \geq 0$ for all $i \in \mathbb{N}^{*}$ ).

Then, $\rho_{1} \in L^{p}\left(\Omega_{T}\right)$, for any finite time $T>0$.

Proof We define

$$
a^{I}:=\inf _{i \geq I} d_{i}, \quad \text { and } \quad b^{I}:=\sup _{i \geq I} d_{i}
$$

Since $\left(d_{i}\right)$ converges toward a positive limit, there exist a positive integer $I$ for all $\left.p^{\prime} \in\right] 1,+\infty[$ such that

$$
\frac{b^{I}-a^{I}}{b^{I}+a^{I}} \mathcal{K}_{\frac{a^{I}+b^{I}}{2}, p^{\prime}}<1 .
$$


We then consider

$$
\rho_{1}^{I}:=\sum_{i=I}^{\infty} i c_{i}, \quad \text { and } \quad M_{1}^{I}:=\frac{\sum_{i=I}^{\infty} i d_{i} c_{i}}{\sum_{i=I}^{\infty} i c_{i}}
$$

Note that thanks to Lemma 3.2, it is enough to prove that $\rho_{1}^{I} \in L^{p}\left(\Omega_{T}\right)$ in order to conclude the proof of Lemma 3.3. We therefore compute (remember that $a_{i, j}=a_{j, i}$ )

$$
\begin{aligned}
\partial_{t} \rho_{1}^{I}-\Delta_{x}\left(M_{1}^{I} \rho_{1}^{I}\right) & =\frac{1}{2} \sum_{i=1}^{\infty} \sum_{j=1}^{\infty} a_{i, j} c_{i} c_{j}\left((i+j) \mathbb{1}_{i+j \geq I}-i \mathbb{1}_{i \geq I}-j \mathbb{1}_{j \geq I}\right) \\
& =\frac{1}{2} \sum_{i=1}^{\infty} \sum_{j=1}^{\infty} a_{i, j} c_{i} c_{j}\left(i\left(\mathbb{1}_{i+j \geq I}-\mathbb{1}_{i \geq I}\right)+j\left(\mathbb{1}_{i+j \geq I}-\mathbb{1}_{j \geq I}\right)\right) \\
& =\sum_{i=1}^{\infty} \sum_{j=1}^{\infty} a_{i, j} c_{i} c_{j} i\left(\mathbb{1}_{i+j \geq I}-\mathbb{1}_{i \geq I}\right) .
\end{aligned}
$$

Next, by using assumption (15), and more precisely that $a_{i, j} \leq C\left(i^{\gamma}+j^{\gamma}\right) \leq$ $C(i+j)$, we have

$$
\begin{aligned}
\sum_{i=1}^{\infty} \sum_{j=1}^{\infty} a_{i, j} c_{i} c_{j} i\left(\mathbb{1}_{i+j \geq I}-\mathbb{1}_{i \geq I}\right) & \leq C \sum_{i=1}^{\infty} \sum_{j=1}^{\infty}(i+j) c_{i} c_{j} i\left(\mathbb{1}_{i+j \geq I}-\mathbb{1}_{i \geq I}\right) \\
& \leq C \sum_{i=1}^{I-1} \sum_{j=1}^{\infty} i^{2} c_{i} c_{j}+C \sum_{i=1}^{I-1} \sum_{j=1}^{\infty} i c_{i} j c_{j} \\
& \leq 2 C\left(\sum_{i=1}^{I-1} i^{2} c_{i}\right)\left(\sum_{j=1}^{\infty} j c_{j}\right) .
\end{aligned}
$$

Thus, we obtain

$$
\partial_{t} \rho_{1}^{I}-\Delta_{x}\left(M_{1}^{I} \rho_{1}^{I}\right) \leq \psi_{1} \rho_{1}^{I}+\psi_{2}
$$

where $\psi_{1}=2 C \sum_{i=1}^{I-1} i^{2} c_{i}$, and $\psi_{2}=\psi_{1} \sum_{i=1}^{I-1} j c_{j}$. Now thanks to Lemma 3.2, both $\psi_{1}$ and $\psi_{2}$ belong to $L^{\infty}\left(\Omega_{T}\right)$. Then, if we denote (for $\left.i \in\{1,2\}\right), \mu_{i}:=$ $\left\|\psi_{i}\right\|_{L^{\infty}\left(\Omega_{T}\right)}$, we get

$$
\partial_{t} \rho_{1}^{I}-\Delta_{x}\left(M_{1}^{I} \rho_{1}^{I}\right) \leq \mu_{1} \rho_{1}^{I}+\mu_{2},
$$

and we can conclude using Proposition 2.5.

Remark 3.4 Note that aside from symmetry, the above proof only requires the estimate $a_{i, j} \leq C i j$, which is a much weaker restriction on the coagulation coefficients than the "sublinear" assumption (15). However, "strictly superlinear" coagulation is known to 
produce gelation already in the spatially homogeneous case. Nonetheless, it is known for the homogeneous case that adding sufficiently strong fragmentation in the model can prevent gelation even with "superlinear" coagulation (see for instance $[7,8]$ ). Similar results in presence of diffusion, together with generalisations of some results of this paper to models including fragmentation are discussed in [3].

Continuation of the proof of Theorem 1.6. We shall now prove the propagation of $L^{p}$, $(p<+\infty)$ regularity for moments of higher order. This is done still under the assumption that the diffusion coefficients $d_{i}$ converge towards a strictly positive limit.

We first introduce

$$
M_{k}^{I}:=\frac{\sum_{i=I}^{\infty} i^{k} d_{i} c_{i}}{\sum_{i=I}^{\infty} i^{k} c_{i}}, \quad a^{I} \leq M_{k}^{I} \leq b^{I} .
$$

The proof of propagation for moments of higher order involves the a priori estimate established in Proposition 2.6. Therefore the results of Theorem 1.6 (for $k>1$ ) only apply to such solutions, which are constructed as a limit of solutions of a truncated system. For a clearer exposition of the proof, we first perform the computations formally and then show afterwards how to conclude rigorously through the use of the truncated systems (9), (10).

We proceed by induction and assume that (for some integer $k$ ), $\rho_{l} \in L^{p}\left(\Omega_{T}\right)$, for all $p<+\infty$ and every $l \leq k-1$. Note that Lemma 3.3 ensures that the induction hypothesis holds for $k=2$. For any $I \in \mathbb{N}^{*}$ (using (15)), we have $\rho_{k}^{I}=\sum_{i=I}^{\infty} i^{k} c_{i}$

$$
\begin{aligned}
\partial_{t} \rho_{k}^{I}-\Delta_{x}\left(M_{k}^{I} \rho_{k}^{I}\right) \leq & \frac{1}{2} \sum_{i=1}^{\infty} \sum_{j=1}^{\infty} a_{i, j} c_{i} c_{j}\left((i+j)^{k} \mathbb{1}_{i+j \geq I}-i^{k} \mathbb{1}_{i \geq I}-j^{k} \mathbb{1}_{j \geq I}\right) \\
\leq & \frac{C}{2} \sum_{l=1}^{k-1}\left(\begin{array}{l}
k \\
l
\end{array}\right) \sum_{i=1}^{\infty} \sum_{j=1}^{\infty}\left(i^{\gamma}+j^{\gamma}\right) i^{l} c_{i} j^{k-l} c_{j} \\
& +\frac{C}{2} \sum_{i=1}^{\infty} \sum_{j=1}^{\infty}\left(i^{\gamma}+j^{\gamma}\right) c_{i} c_{j}\left(i^{k}\left(\mathbb{1}_{i+j \geq I}-\mathbb{1}_{i \geq I}\right)\right. \\
& \left.+j^{k}\left(\mathbb{1}_{i+j \geq I}-\mathbb{1}_{j \geq I}\right)\right) \\
\leq & C \sum_{l=1}^{k-1}\left(\begin{array}{l}
k \\
l
\end{array}\right) \sum_{i=1}^{\infty} i^{\gamma+l} c_{i} \sum_{j=1}^{\infty} j^{k-l} c_{j} \\
& +C \sum_{i=1}^{I-1} i^{\gamma+k} c_{i} \sum_{j=1}^{\infty} c_{j}+C \sum_{i=1}^{I-1} i^{k} c_{i} \sum_{j=1}^{\infty} j^{\gamma} c_{j}
\end{aligned}
$$

We point out that an alternative way of getting (26), maybe more reminiscent of some earlier computations (see [7]), would be to use that

$$
\left(i^{\gamma}+j^{\gamma}\right)\left((i+j)^{k}-i^{k}-j^{k}\right) \leq C(k)\left(i^{k+\gamma-1} j+j^{k+\gamma-1} i\right) .
$$


In the special case $k=2$, (26) yields (using $\gamma \leq 1$ )

$$
\partial_{t} \rho_{2}^{I}-\Delta_{x}\left(M_{2}^{I} \rho_{2}^{I}\right) \leq 2 C \rho_{1+\gamma} \rho_{1}+2 C \rho_{1} \sum_{i=1}^{I-1} i^{3} c_{i}
$$

Moreover, we can split the moment of order $1+\gamma$ between a finite part that we already control and a tail, and then bound the latter using Hölder's inequality:

$\rho_{1+\gamma} \leq \sum_{i=1}^{I-1} i^{1+\gamma} c_{i}+\rho_{1+\gamma}^{I} \leq \sum_{i=1}^{I-1} i^{2} c_{i}+\left(\rho_{2}^{I}\right)^{1-\varepsilon}\left(\rho_{1}^{I}\right)^{\varepsilon}, \quad$ where $\varepsilon=1-\gamma>0$

Therefore, we end up with

$$
\partial_{t} \rho_{2}^{I}-\Delta_{x}\left(M_{2}^{I} \rho_{2}^{I}\right) \leq 2 C\left(\rho_{2}^{I}\right)^{1-\varepsilon}\left(\rho_{1}\right)^{1+\varepsilon}+4 C \rho_{1} \sum_{i=1}^{I-1} i^{3} c_{i}
$$

and the last term in the r.h.s. of (27) lies in $L^{p}\left(\Omega_{T}\right)$ for every $p<+\infty$ thanks to Lemmas 3.2 and 3.3. Thus, taking $I$ large enough, inequality (27) and Proposition 2.6 formally yield a (computable) bound for $\rho_{2}^{I}$ (and therefore $\rho_{2}$ ) in $L^{p}\left(\Omega_{T}\right)$, for all $p<+\infty$.

Next, returning to the general case of moments of order $k>2$, we estimate (26) as

$$
\begin{aligned}
\partial_{t} \rho_{k}^{I}-\Delta_{x}\left(M_{k}^{I} \rho_{k}^{I}\right) \leq & k C \sum_{i=1}^{\infty} i^{\gamma+k-1} c_{i} \sum_{j=1}^{\infty} j c_{j}+C \sum_{l=1}^{k-2}\left(\begin{array}{l}
k \\
l
\end{array}\right) \sum_{i=1}^{\infty} i^{\gamma+l} c_{i} \sum_{j=1}^{\infty} j^{k-l} c_{j} \\
& +C \sum_{i=1}^{I-1} i^{\gamma+k} c_{i} \sum_{j=1}^{\infty} c_{j}+C \sum_{i=1}^{I-1} i^{k} c_{i} \sum_{j=1}^{\infty} j^{\gamma} c_{j} \\
\leq & k C \rho_{k-1+\gamma} \rho_{1}+C \sum_{l=1}^{k-2}\left(\begin{array}{l}
k \\
l
\end{array}\right) \rho_{l+1} \rho_{k-l}+2 C \rho_{1} \sum_{i=1}^{I-1} i^{k+1} c_{i},
\end{aligned}
$$

where we used $\gamma \leq 1$. Again, we can split the moment of order $k-1+\gamma$ between a finite part that we already control and a tail, and then bound the latter using Hölder's inequality:

$$
\begin{aligned}
\rho_{k-1+\gamma} \leq & \sum_{i=1}^{I-1} i^{k-1+\gamma} c_{i}+\rho_{k-1+\gamma}^{I} \leq \sum_{i=1}^{I-1} i^{k} c_{i}+\left(\rho_{k}^{I}\right)^{1-\varepsilon}\left(\rho_{1}^{I}\right)^{\varepsilon}, \\
\text { where } & \varepsilon=\frac{1-\gamma}{k-1}>0 .
\end{aligned}
$$


Therefore, we end up with

$\partial_{t} \rho_{k}^{I}-\Delta_{x}\left(M_{k}^{I} \rho_{k}^{I}\right) \leq k C\left(\rho_{k}^{I}\right)^{1-\varepsilon}\left(\rho_{1}\right)^{1+\varepsilon}+C \sum_{l=1}^{k-2}\left(\begin{array}{l}k \\ l\end{array}\right) \rho_{l+1} \rho_{k-l}+(k+2) C \rho_{1} \sum_{i=1}^{I-1} i^{k+1} c_{i}$,

and the last two terms in the r.h.s. of (28) lie in $L^{p}\left(\Omega_{T}\right)$ for every $p<+\infty$ thanks to Lemmas 3.2, 3.3 and the induction hypothesis. Thus, taking $I$ large enough, inequality (28) and Proposition 2.6 would yield a (computable) bound for $\rho_{k}^{I}$ (and therefore $\rho_{k}$ ) in $L^{p}\left(\Omega_{T}\right)$, for all $p<+\infty$, except that Proposition 2.6 also requires to a priori know that $\rho_{k}^{I} \in L^{p}\left(\Omega_{T}\right)$, which is not the case at this point.

In order to make the proof rigorous, we need to apply Proposition 2.6 to smooth solutions obtained by truncating the original system. Therefore, for an integer $n>I$, we consider $c^{n}=\left(c_{i}^{n}, \ldots, c_{n}^{n}\right)$ the solution of (9), (10) and define

$$
\rho_{k}^{n}=\sum_{i=1}^{n} i^{k} c_{i}^{n}, \quad \rho_{k}^{n, I}=\sum_{i=I}^{n} i^{k} c_{i}^{n} \quad \text { and } \quad M_{k}^{n, I}=\frac{\sum_{i=I}^{n} i^{k} d_{i} c_{i}^{n}}{\sum_{i=I}^{n} i^{k} c_{i}^{n}}
$$

We then perform the same computations as previously, taking into account the truncation in the coagulation kernel (10). We get for the second order moment (and $n>I)$

$$
\begin{aligned}
\partial_{t} \rho_{2}^{n, I}-\Delta_{x}\left(M_{2}^{n, I} \rho_{2}^{n, I}\right) & \leq 2 C\left(\rho_{2}^{n, I}\right)^{1-\varepsilon}\left(\rho_{1}^{n}\right)^{1+\varepsilon}+4 C \rho_{1}^{n} \sum_{i=1}^{I-1} i^{3} c_{i}^{n} \\
& =A_{1}^{n}\left(\rho_{2}^{n, I}\right)^{1-\varepsilon}+B_{1}^{n, I},
\end{aligned}
$$

where $\varepsilon=1-\gamma>0$ and $A_{1}^{n}$ and $B_{1}^{n, I}$ only depend on the approximating first order moment $\rho_{1}^{n}$ and on a finite number of approximate concentrations $c_{i}^{n}$, for $i<I$. Since this time we know that $\rho_{2}^{n, I} \in L^{p}\left(\Omega_{T}\right)$, we can apply Proposition 2.6 and get the estimate

$$
\begin{aligned}
\int_{\Omega_{T}}\left(\rho_{2}^{n, I}\right)^{p} & \leq C\left(\int_{\Omega}\left(\rho_{2}^{n, I}(0)\right)^{p}+\int_{\Omega_{T}}\left(A_{1}^{n}\right)^{\frac{p}{\varepsilon}}+\int_{\Omega_{T}}\left(B_{1}^{n, I}\right)^{p}\right) \\
& \leq C\left(\int_{\Omega}\left(\rho_{2}^{i n}\right)^{p}+\int_{\Omega_{T}}\left(A_{1}^{n}\right)^{\frac{p}{\varepsilon}}+\int_{\Omega_{T}}\left(B_{1}^{n, I}\right)^{p}\right),
\end{aligned}
$$

where the constant $C$ does not depend on $n$. In order to complete the proof, we still have to show that $A_{1}^{n}$ and $B_{1}^{n, I}$ can be bounded in $L^{p}$ norms uniformly-in- $n$.

Prior to that, we consider also the approximation of any general moments of order $k>2$. By estimating as in the computation leading to (28), we obtain for the truncated moments of order $k>2$ 


$$
\begin{aligned}
\partial_{t} \rho_{k}^{n, I}-\Delta_{x}\left(M_{k}^{n, I} \rho_{k}^{n, I}\right) \leq & k C\left(\rho_{k}^{n, I}\right)^{1-\varepsilon}\left(\rho_{1}^{n}\right)^{1+\varepsilon} \\
& +C \sum_{l=1}^{k-2}\left(\begin{array}{l}
k \\
l
\end{array}\right) \rho_{l+1}^{n} \rho_{k-l}^{n}+(k+2) C \rho_{1}^{n} \sum_{i=1}^{I-1} i^{k+1} c_{i}^{n} \\
= & A_{k-1}^{n}\left(\rho_{k}^{n, I}\right)^{1-\varepsilon}+B_{k-1}^{n, I},
\end{aligned}
$$

with $\varepsilon=\frac{1-\gamma}{k-1}>0$, and where $A_{k-1}^{n}$ and $B_{k-1}^{n, I}$ only depend on moments $\rho_{l}^{n}$ of integer order $l$ between 1 and $k-1$ and on a finite number of concentrations $c_{i}^{n}$ (for $i<I$ ). Moreover, since $\rho_{k}^{n, I} \in L^{p}\left(\Omega_{T}\right)$, we can again apply Proposition 2.6 and estimate

$$
\int_{\Omega_{T}}\left(\rho_{k}^{n, I}\right)^{p} \leq C\left(\int_{\Omega}\left(\rho_{k}^{i n}\right)^{p}+\int_{\Omega_{T}}\left(A_{k-1}^{n}\right)^{\frac{p}{\varepsilon}}+\int_{\Omega_{T}}\left(B_{k-1}^{n, I}\right)^{p}\right),
$$

where the constant $C$ does not depend on $n$. So we again have to prove that $A_{k-1}^{n}$ and $B_{k-1}^{n, I}$ can be bounded in $L^{p}$ norms uniformly-in- $n$.

First we notice that for any given $i$, since $c_{i}^{i n} \in L^{\infty}(\Omega)$, the concentrations $c_{i}^{n}$ can be bounded in $L^{\infty}\left(\Omega_{T}\right)$ uniformly-in- $n$ by the computations of Lemma 3.2. Indeed,

$$
\partial_{t} c_{1}^{n}-d_{1} \Delta_{x} c_{1}^{n} \leq Q_{1}^{+, n}\left(c^{n}\right)=0
$$

yields a uniform-in- $n$ bound for $c_{1}^{n}$, and then

$$
\partial_{t} c_{i}^{n}-d_{i} \Delta_{x} c_{i}^{n} \leq \frac{1}{2} \sum_{j=1}^{i-1} a_{i-j, j} c_{i-j}^{n} c_{j}^{n}
$$

allows to conclude inductively in $i$ for all $T>0$. Now, from that fact (that is, each $c_{i}^{n}$ is uniformly-in- $n$ bounded in $L^{\infty}\left(\Omega_{T}\right)$ ), we get that $\rho_{1}^{n, I}$ (and also $\rho_{1}^{n}$ ) is uniformly-in- $n$ bounded in any $L^{p}$ norm, $p<+\infty$, thanks to Lemma 3.3 and Proposition 2.5 (one just needs to repeat the computations in the proof of Lemma 3.3 with $\rho_{1}^{n, I}$ instead of $\rho_{1}^{I}$ ). Therefore $A_{1}^{n}$ and $B_{1}^{n, I}$ are uniformly-in- $n$ bounded in any $L^{p}\left(\Omega_{T}\right), p<+\infty$, and going back to (29), this yields that $\rho_{2}^{n, I}$ (and thus $\rho_{2}^{n}$ ) is uniformly-in- $n$ bounded in any $L^{p}\left(\Omega_{T}\right), p<+\infty$. Similarly, we can prove inductively using (30) that for all $k>2$, $\rho_{k}^{n, I}$ (and thus $\rho_{k}^{n}$ ) is uniformly-in- $n$ bounded in any $L^{p}\left(\Omega_{T}\right), p<+\infty$. Applying Fatou's Lemma we can conclude that, for the weak solutions given by Proposition 1.2, $\rho_{k}$ is bounded in any $L^{p}\left(\Omega_{T}\right), p<+\infty$.

Remark 3.5 Fatou's Lemma is enough here to show that $\rho_{k} \in L^{p}\left(\Omega_{T}\right)$, but since for any $k \in \mathbb{N}^{*}$ we know that $\rho_{k+1}^{n}$ is bounded in $L^{p}\left(\Omega_{T}\right)$ uniformly-in- $n$, we could show by interpolation that we do in fact have the convergence of $\rho_{k}^{n}$ to $\rho_{k}$ in $L^{p}\left(\Omega_{T}\right)$.

Remark 3.6 Note that Theorem 1.6 states that we have propagation of the moment $\rho_{k}$ in every $L^{p}\left(\Omega_{T}\right), p<+\infty$, provided that the initial moment $\rho_{k}^{i n}$ lies in every $L^{p}(\Omega)$, 
$p<+\infty$. If we only want to get propagation of $\rho_{k}$ in $L^{p}\left(\Omega_{T}\right)$ for some fixed $p$, we can relax a bit the hypothesis, but to apply the above proof we still need to assume that initial moment of lower order $\rho_{l}^{i n}, l<k$, are in some space $L^{q}(\Omega)$ with $q>p$ depending of the magnitude of the coagulation. For instance, if we want to get for some fixed $p$ that $\rho_{2} \in L^{p}\left(\Omega_{T}\right)$ with the method of Theorem 1.6, we need to assume that $\rho_{2}^{i n} \in L^{p}(\Omega)$ and $\rho_{1}^{i n} \in L^{q}(\Omega)$, where $q=\frac{2-\gamma}{1-\gamma} p$.

Remark 3.7 Finally, we point out that Theorem 1.6 would still hold if a finite number of diffusion coefficients $d_{i}$ were equal to 0 . Indeed Lemma 3.2 is still valid in this case: if $c_{j} \in L^{\infty}\left(\Omega_{T}\right)$ for all $j<i$ and $d_{i}=0$, then

$$
\partial_{t} c_{i} \leq Q_{i}^{+}(c)=\frac{1}{2} \sum_{j=1}^{i-1} a_{i, j} c_{i-j} c_{j}
$$

shows that $c_{i} \in L^{\infty}\left(\Omega_{T}\right)$; and up to taking $I$ large enough, we would still get $\inf _{i \geq I} d_{i}>0$, so we could still apply Propositions 2.5 and 2.6 to control the tail moments $\rho_{k}^{I}$.

\section{Propagation of Sobolev norms for moments}

In this Section, we show how the parabolic structure of equation (1) can be used to improve the results of Theorem 1.6 and get higher regularity as stated in Theorem 1.9. We also explain how the obtained regularity in fact implies uniqueness. For some early work on the diffusive coagulation-fragmentation equation, using extensively the parabolic structure, we refer the reader to [2].

Proof of Theorem 1.9. We consider a solution provided by Theorem 1.6, for which we already know that we have propagation of moments in $L^{p}$ spaces. Remembering (1), we want to use the properties of the heat equation to get additional regularity, and to do so we first need to estimate the coagulation term. This is the content of the following lemma.

Lemma 4.1 Let $\Omega$ be a smooth bounded domain of $\mathbb{R}^{N}$ and $s \in \mathbb{N}$. Assume that $\left(c_{i}\right)_{i \in \mathbb{N}^{*}}$ is a sequence of positive functions defined on $\Omega_{T}$ such that

$$
\sup _{i \geq 1}\left\|i^{k} c_{i}\right\|_{W^{s, p}\left(\Omega_{T}\right)}<+\infty, \quad \forall k \in \mathbb{N}, \forall p<+\infty .
$$

Then, assuming (2) and (15), the following estimates hold:

$$
\sup _{i \geq 1}\left\|i^{k} Q_{i}(c)\right\|_{W^{s, p}\left(\Omega_{T}\right)}<+\infty, \quad \forall k \in \mathbb{N}, \forall p<+\infty .
$$

Remark 4.2 We remark that analog statements to (31) and (32) could be made in terms of fractional Sobolev spaces, for instance by interpolation arguments. 
Proof Remembering (2) and using the sublinearity of the coagulation coefficients (15) $\left(a_{i, j} \leq C\left(i^{\gamma}+j^{\gamma}\right) \leq C(i+j) \leq 2 C i j\right)$, we estimate

$$
\left\|i^{k} Q_{i}(c)\right\|_{W^{s, p}\left(\Omega_{T}\right)} \leq C \sum_{j=1}^{i-1} i^{k+1}\left\|c_{i-j} c_{j}\right\|_{W^{s, p}\left(\Omega_{T}\right)}+2 C\left\|i^{k+1} c_{i} \sum_{j=1}^{\infty} j c_{j}\right\|_{W^{s, p}\left(\Omega_{T}\right)} .
$$

We now use the algebra property of $\bigcap_{p<+\infty} W^{s, p}\left(\Omega_{T}\right)$. More precisely, by combining Cauchy-Schwarz's inequality and Leibniz's formula, the following estimate holds:

$$
\|u v\|_{W^{s, p}\left(\Omega_{T}\right)} \leq C(s)\|u\|_{W^{s, 2 p}\left(\Omega_{T}\right)}\|v\|_{W^{s, 2 p}\left(\Omega_{T}\right)},
$$

where $C(s)$ is a constant depending only on $s$. Therefore,

$$
\begin{aligned}
& \left\|i^{k} Q_{i}(c)\right\|_{W^{s, p}\left(\Omega_{T}\right)} \\
& \leq C(s)\left(\sum_{j=1}^{i-1} i^{k+1}\left\|c_{i-j}\right\|_{W^{s, 2 p}\left(\Omega_{T}\right)}\left\|c_{j}\right\|_{W^{s, 2 p}\left(\Omega_{T}\right)}\right. \\
& \left.\quad+\left\|i^{k+1} c_{i}\right\|_{W^{s, 2 p}\left(\Omega_{T}\right)} \sum_{j=1}^{\infty}\left\|j c_{j}\right\|_{W^{s, 2 p}\left(\Omega_{T}\right)}\right) \\
& =C(s)\left(\sum_{j=1}^{i-1} \frac{i^{k+1}}{(i-j)^{k+2} j^{k+2}\left\|(i-j)^{k+2} c_{i-j}\right\|_{W^{s, 2 p}\left(\Omega_{T}\right)}\left\|j^{k+2} c_{j}\right\|_{W^{s, 2 p}\left(\Omega_{T}\right)}}\right. \\
& \left.\quad+\left\|i^{k+1} c_{i}\right\|_{W^{s, 2 p}\left(\Omega_{T}\right)} \sum_{j=1}^{\infty} \frac{1}{j^{2}}\left\|j^{3} c_{j}\right\|_{W^{s, 2 p}\left(\Omega_{T}\right)}\right) .
\end{aligned}
$$

Using (31), we get

$$
\left\|i^{k} Q_{i}(c)\right\|_{W^{s, p}\left(\Omega_{T}\right)} \leq C(s, p, k)\left(1+\sum_{j=1}^{i-1} \frac{i^{k+1}}{(i-j)^{k+2} j^{k+2}}\right)
$$

where $C(s, p, k)$ depends on the quantities $\sup _{j \geq 1}\left\|j^{l} c_{j}\right\|_{W^{s, 2 p}\left(\Omega_{T}\right)}$ for $l \in \mathbb{N}$, but not on $i$. We then show that (for any $k \in \mathbb{N}$ )

$$
\sup _{i \geq 1} \sum_{j=1}^{i-1} \frac{i^{k+1}}{(i-j)^{k+2} j^{k+2}}<\infty
$$


Indeed, by symmetry, we know that (denoting by $[m]$ the integer part of $m$ )

$$
\begin{aligned}
\sup _{i \geq 1} \sum_{j=1}^{i-1} \frac{i^{k+1}}{j^{k+2}(i-j)^{k+2}} & \leq 2 \sup _{i \geq 1} \sum_{j=1}^{\left[\frac{i}{2}\right]} \frac{i^{k+1}}{j^{k+2}(i-j)^{k+2}} \leq 2 \sup _{i \geq 1} \sum_{j=1}^{\left[\frac{i}{2}\right]}\left(\frac{i}{i-\left[\frac{i}{2}\right]}\right)^{k+2} \frac{1}{j^{k+2}} \\
& \leq 2^{k+3} \sum_{j=1}^{\infty} \frac{1}{j^{k+2}}<\infty .
\end{aligned}
$$

This implies that

$$
\sup _{i \geq 1}\left\|i^{k} Q_{i}(c)\right\|_{W^{s, p}\left(\Omega_{T}\right)}<+\infty, \quad \forall k \in \mathbb{N}, \quad \forall p<+\infty
$$

and Lemma 4.1 is proven.

Continuation of the proof of Theorem 1.9. Now, we can show that under the hypothesis of Theorem 1.9, the concentrations $\left(c_{i}\right)$ considered in Theorem 1.6 satisfy

$$
\sup _{i \geq 1}\left\|i^{k} c_{i}\right\|_{W^{s, p}\left(\Omega_{T}\right)}<+\infty, \quad \forall k \in \mathbb{N}, \quad \forall p<+\infty, \forall s \in \mathbb{N} .
$$

We shall prove (33) by induction on $s$. The case $s=0$ is a direct consequence of Theorem 1.6. Then, if for some $s \in \mathbb{N}$,

$$
\sup _{i \geq 1}\left\|i^{k} c_{i}\right\|_{W^{s, p}\left(\Omega_{T}\right)}<+\infty, \quad \forall k \in \mathbb{N}, \quad \forall p<+\infty,
$$

we see that Lemma 4.1 yields the estimate

$$
\sup _{i \geq 1}\left\|i^{k} Q_{i}(c)\right\|_{W^{s, p}\left(\Omega_{T}\right)}<+\infty, \quad \forall k \in \mathbb{N}, \quad \forall p<+\infty .
$$

Remembering that $i^{k} c_{i}$ satisfies

$$
\begin{cases}\left(\partial_{t}-d_{i} \Delta_{x}\right) i^{k} c_{i}=i^{k} Q_{i}(c) & \text { on } \Omega_{T} \\ \nabla_{x}\left(i^{k} c_{i}\right) \cdot v=0 & \text { on }[0, T] \times \partial \Omega \\ i^{k} c_{i}(0, \cdot)=i^{k} c_{i}^{i n} & \text { on } \Omega,\end{cases}
$$

and using the regularising properties of the heat equation (they can be used uniformly w.r.t. $i$ since the diffusion rates $d_{i}$ are bounded above and below by strictly positive constants), we get the estimate

$$
\sup _{i \geq 1}\left\|i^{k} c_{i}\right\|_{W^{s+1, p}\left(\Omega_{T}\right)}<+\infty, \quad \forall k \in \mathbb{N}, \quad \forall p<+\infty .
$$


This concludes the proof of (33). Notice that we also get $W^{s, p}$ estimates for polynomial moments of any order, because

$$
\left\|\rho_{k}\right\|_{W^{s, p}\left(\Omega_{T}\right)} \leq \sum_{i=1}^{\infty} \frac{1}{i^{2}}\left\|i^{k+2} c_{i}\right\|_{W^{s, p}\left(\Omega_{T}\right)} \leq \sup _{i \geq 1}\left\|i^{k+2} c_{i}\right\|_{W^{s, p}\left(\Omega_{T}\right)} \sum_{i=1}^{\infty} \frac{1}{i^{2}} .
$$

The $\mathcal{C}^{\infty}$ regularity announced in Theorem 1.9 is now a straightforward consequence of Sobolev embeddings [note that while $\Omega$ is assumed to be smooth, $\left.\Omega_{T}=\Omega \times\right] 0, T$ [ will be only of Lipschitz regularity,but this is enough to apply the required Sobolev embeddings (see for instance [1])]. The uniqueness of such a smooth solution is given by a straightforward extension of [15, Theorem 1.4] to the case of bounded smooth domains with Neumann boundary conditions (the uniqueness Theorem of [15] is stated when the spatial domain is $\mathbb{R}^{N}$ ), where it is proven that there cannot exist more than one weak solution to (1), (2) satisfying $\rho_{2} \in L^{\infty}\left(\Omega_{T}\right)$, as soon as the coagulation coefficients satisfy $a_{i, j} \leq C i j$, which is implied by (15).

Acknowledgements Open access funding provided by University of Graz. The research leading to this paper was partially funded by the french "ANR blanche" project Kibord: ANR-13-BS01-0004. K.F. was partially supported by NAWI Graz and acknowledges the kind hospitality of the ENS Cachan. The research leading to this paper was also funded by Université Sorbonne Paris Cité, in the framework of the "Investissements d'Avenir", convention ANR-11-IDEX-0005.

Open Access This article is distributed under the terms of the Creative Commons Attribution 4.0 International License (http://creativecommons.org/licenses/by/4.0/), which permits unrestricted use, distribution, and reproduction in any medium, provided you give appropriate credit to the original author(s) and the source, provide a link to the Creative Commons license, and indicate if changes were made.

\section{Appendix}

This section is devoted to the proof of the existence of solutions of dual problems such as

$$
\begin{cases}\partial_{t} w+M \Delta_{x} w=f & \text { on } \Omega_{T}, \\ \nabla_{x} w \cdot v=0 & \text { on }] 0, T[\times \partial \Omega, \\ w(T, \cdot)=0 & \text { on } \Omega,\end{cases}
$$

when $f$ lies in some $L^{q}\left(\Omega_{T}\right)$, provided that there exist constants $0<a \leq b$ (sufficiently close from one another in the sense of hypothesis (17)) such that $a \leq M \leq b$. We emphasise that $M:=M(t, x)$ is not assumed to be continuous.

Note that with the change of time variable $\tau=T-t$, the above dual problem becomes a forward heat equation with homogeneous initial data:

$$
\begin{cases}\partial_{t} v-M \Delta_{x} v=-f & \text { on } \Omega_{T} \\ \nabla_{x} v \cdot v=0 & \text { on }] 0, T[\times \partial \Omega \\ v(0, \cdot)=0 & \text { on } \Omega\end{cases}
$$

In the case of parabolic equations in divergence form, i.e. when

$$
\partial_{t} v-\operatorname{div}_{x}\left(A(t, x) \nabla_{x} v\right)=f
$$


it is well known that the strict ellipticity property

$$
\xi^{\mathrm{T}} A(t, x) \xi \geq \lambda|\xi|^{2}, \quad \lambda>0, \quad \forall(t, x) \in \Omega_{T}, \quad \forall \xi \in \mathbb{R}^{N},
$$

guarantees the existence of weak solutions even if $A$ is only in $L^{\infty}$, see e.g. [17].

However, we are here interested in parabolic equations in non-divergence form, i.e.

$$
\partial_{t} v-\sum_{i, j} A_{i, j}(t, x) \partial_{i, j}^{2} v=f,
$$

and we recall that when $A(t, x)=\left(A_{i, j}(t, x)\right)_{i, j}$ is not smooth, the two formulations (35) and (36) are not equivalent.

Unfortunately, the existence theory for parabolic equations with discontinuous coefficients is much less developed in the non-divergence case (36) than in the divergence case (35), and some additional assumptions on $A$ (that is, stronger than strict ellipticity) are needed.

One class of available results for parabolic equations in non-divergence form assumes coefficients which are VMO in at least sufficiently many of the time/space variables, see e.g. $[14,16]$ for references which consider $f \in L^{q}\left(\Omega_{T}\right)$ with $1<q<2$.

Another approach, in [21], consist in treating equation (36) as a perturbation of the standard heat equation

$$
\partial_{t} v-\Delta_{x} v=f
$$

and existence of a unique weak solution is then proven under the so called Cordes condition

$$
\left\|\frac{\sum_{1 \leq i, j \leq N} A_{i, j}^{2}+1}{\left(\sum_{1 \leq i \leq N} a_{i, i}+1\right)^{2}}\right\|_{L^{\infty}\left(\Omega_{T}\right)}<\frac{1}{N},
$$

which in some sense measures how far $A$ is from the identity matrix and explicitly involves the dimension $N$.

The equation appearing in (34) is less general than the one treated in [21], since we only consider matrices $A$ of the form

$$
A_{i, j}(t, x)=\delta_{i, j} M(t, x)
$$

but unfortunately assumption (37) may not be satisfied. We can however adapt the techniques of [21] to get a proof of existence (and uniqueness) under our assumptions on $M$. The main idea consists in considering $\partial_{t}-M \Delta_{x}$ as a perturbation of $\partial_{t}-m \Delta_{x}$ (instead of $\partial_{t}-\Delta_{x}$ ), where $m$ can be seen as a mean value of $M$. This is the content of the following:

Proposition 4.3 Let $\Omega$ be a bounded smooth subset of $\mathbb{R}^{N}$ and consider $M: \Omega_{T} \rightarrow$ $\mathbb{R}_{+}$satisfying $a \leq M \leq b$ for some $a, b>0$, and $f \in L^{q}\left(\Omega_{T}\right)$. Assume that the closeness condition (17) holds. 
Then, there exists a unique $u \in L^{q}(] 0, T\left[; W^{2, q}(\Omega)\right) \cap W^{1, q}(] 0, T\left[; L^{q}(\Omega)\right)$ such that

$$
\begin{cases}\partial_{t} u-M \Delta_{x} u=f & \text { on } \Omega_{T} \\ \nabla_{x} u \cdot v=0 & \text { on }] 0, T[\times \partial \Omega \\ u(0, \cdot)=0 & \text { on } \Omega\end{cases}
$$

Proof We first rewrite the equation as a perturbation of a heat equation with constant diffusion coefficient:

$$
\partial_{t} u-m \Delta_{x} u=-(m-M) \Delta_{x} u+f
$$

where $m=\frac{a+b}{2}$. Then we introduce the space

$$
Z^{q}:=\left\{v \in L^{q}(] 0, T\left[; W^{2, q}(\Omega)\right) \cap W^{1, q}(] 0, T\left[; L^{q}(\Omega)\right), \nabla_{x} v \cdot v=0, v(0, \cdot)=0\right\},
$$

and the operator $F$ defined on $Z^{q}$, which associates to each $v \in Z^{q}$ the unique solution $F v \in Z^{q}$ of

$$
\begin{cases}\partial_{t}(F v)-m \Delta_{x}(F v)=-(m-M) \Delta_{x} v+f & \text { on } \Omega_{T}, \\ \nabla_{x}(F v) \cdot v=0 & \text { on }[0, T] \times \partial \Omega, \\ (F v)(0, \cdot)=0 & \text { on } \Omega .\end{cases}
$$

Proving Proposition 4.3 is now equivalent to showing the existence of a unique fixed point for $F$. We endow $Z^{q}$ with the norm

$$
\|v\|_{Z_{m}^{q}\left(\Omega_{T}\right)}=\left(\int_{\Omega_{T}}\left|\partial_{t} v\right|^{q}+m^{q} \int_{\Omega_{T}}\left|\Delta_{x} v\right|^{q}\right)^{\frac{1}{q}},
$$

which makes $Z^{q}$ a Banach space. Note that this is indeed a norm on $Z^{q}$ thanks to Calderon-Zygmund inequality:

$$
\int_{\Omega_{T}}\left|D_{x}^{2} v\right|^{q} \leq C \int_{\Omega_{T}}\left|\Delta_{x} v\right|^{q}
$$

We now show that $F$ is a contraction on $\left(Z^{q},\|\cdot\|_{Z_{m}^{q}}\right)$, which will yield the existence of a unique fixed point by the contraction mapping Theorem. For any $v, w \in Z^{q}$, we have

$$
\partial_{t}(F v-F w)-m \Delta_{x}(F v-F w)=-(m-M) \Delta_{x}(v-w),
$$

so that remembering Definition 1.3,

$$
\begin{aligned}
\|F v-F w\|_{Z_{m}^{q}\left(\Omega_{T}\right)} & \leq \frac{b-a}{2} \mathcal{K}_{m, q}\left\|\Delta_{x}(v-w)\right\|_{L^{q}\left(\Omega_{T}\right)} \\
& \leq \frac{b-a}{2} \frac{\mathcal{K}_{m, q}}{m}\|v-w\|_{Z_{m}^{q}\left(\Omega_{T}\right)}
\end{aligned}
$$


Thus, thanks to $m=\frac{a+b}{2}$ and assumption (17), $F$ is a contraction.

Note that in the Hilbert space case $q=2$, it is easily possible (see e.g. [23]) to obtain an explicit bound on $\mathcal{K}_{m, 2}$, namely $\mathcal{K}_{m, 2} \leq 1$, which shows that assumption (17) is always satisfied for $q=2$ (and hence for $q$ sufficiently close to 2, see Remark 1.5). This is the content of the following:

Lemma 4.4 For all $m>0$, we have $\mathcal{K}_{m, 2} \leq 1$, see e.g. [23].

Proof By multiplying

$$
\partial_{t} v-m \Delta_{x} v=f
$$

once by $\partial_{t} v$, once by $-m \Delta_{x} v$, and adding the results, we get

$$
\left(\partial_{t} v\right)^{2}+m^{2}\left(\Delta_{x} v\right)^{2}-2 m \partial_{t} v \Delta_{x} v=f^{2} .
$$

We now show that $\int_{\Omega_{T}} \partial_{t} v \Delta_{x} v \leq 0$. Integrating by parts and using the Neumann boundary conditions and the homogeneous initial data, we see indeed that

$$
\int_{\Omega_{T}} \partial_{t} v \Delta_{x} v=-\int_{0}^{T} \int_{\Omega} \partial_{t} \nabla_{x} v \cdot \nabla_{x} v=-\frac{1}{2} \int_{\Omega}\left|\nabla_{x} v\right|^{2}(T) \leq 0 .
$$

Therefore, we obtain

$$
\int_{\Omega_{T}}\left(\partial_{t} v\right)^{2}+m^{2} \int_{\Omega_{T}}\left(\Delta_{x} v\right)^{2} \leq \int_{\Omega_{T}} f^{2}
$$

so that $\mathcal{K}_{m, 2} \leq 1$.

\section{References}

1. Adams, R.A., Fournier, J.J.F.: Sobolev Spaces, 2nd edn. Academic press, London (2003)

2. Amann, H.: Coagulation-fragmentation processes. Arch. Ration. Mech. Anal. 151, 339-366 (2000)

3. Breden, M.: Applications of improved duality lemmas to the discrete coagulation-fragmentation equations with diffusion. arXiv:1606.07661 (2016) (Preprint)

4. Ball, J.M., Carr, J.: The discrete coagulation-fragmentation equations: existence, uniqueness, and density conservation. J. Stat. Phys. 61(1-2), 203-234 (1990)

5. Canizo, J., Desvillettes, L., Fellner, K.: Regularity and mass conservation for discrete coagulationfragmentation equations with diffusion. Ann. de l'Institut Henri Poincaré (C) Non Linear Anal. 27(2), 639-654 (2010)

6. Canizo, J., Desvillettes, L., Fellner, K.: Improved duality estimates and applications to reactiondiffusion equations. Comm. PDE 39(6), 1185-1204 (2014)

7. Carr, J.: Asymptotic behaviour of solutions to the coagulation-fragmentation equations. I. The strong fragmentation case. Proc. R. Soc. Edinb.: Sect. A Math. 121(3-4), 231-244 (1992)

8. Da Costa, F.P.: Existence and uniqueness of density conserving solutions to the coagulationfragmentation equations with strong fragmentation. J. math. Anal. Appl. 192(3), 892-914 (1995)

9. Desvillettes, L.: About entropy methods for reaction-diffusion equations. Riv. di Mat. dell'Università di Parma 7(7), 81-123 (2007)

10. Desvillettes, L., Fellner, K.: Duality and entropy methods in coagulation-fragmentation models. Rev. di Mat. della Universita di Parma 4(2), 215-263 (2013) 
11. Desvillettes, L., Fellner, K., Pierre, M., Vovelle, J.: Global existence for quadratic systems of reactiondiffusion. Adv. Nonlinear Stud. 7(3), 491-511 (2007)

12. Drake, R.L.: A general mathematical survey of the coagulation equation. In: International Reviews in Aerosol Physics and Chemistry, Oxford, pp. 203-376 (1972)

13. Escobedo, M., Mischler, S., Perthame, B.: Gelation in coagulation and fragmentation models. Commun. Math. Phys. 231(1), 157-188 (2002)

14. Haller-Dintelmann, R., Heck, H., Hieber, M.: $L_{p}-L_{q}$-estimates for parabolic systems in nondivergence form with VMO coefficients. J. London Math. Soc. (2) 74(3), 717-736 (2006)

15. Hammond, A., Rezakhanlou, F.: Moment bounds for the Smoluchowski equation and their consequences. Commun. Math. Phys. 276(3), 645-670 (2007)

16. Krylov, N.V.: Parabolic equations with VMO coefficients in Sobolev spaces with mixed norms. J. Funct. Anal. 250(2), 521-558 (2007)

17. Ladyzenskaja, O.A., Solonnikov, V.A., Uralceva, N.N.: Linear and quasilinear equations of parabolic type. In: Translations of Mathematical Monographs, Vol. 23, American Mathematical Society (1968)

18. Lamberton, D.: Equations d'évolution linéaires associées à des semi-groupes de contractions dans les espaces $L^{p}$. J. Funct. Anal. 72(2), 252-262 (1987)

19. Laurençot, $\mathrm{Ph}$, Mischler, S.: Global existence for the discrete diffusive coagulation-fragmentation equations in $L^{1}$. Rev. Matemática Iberoam. 18(3), 731-745 (2002)

20. Laurençot, Ph., Mischler, S.: On coalescence equations and related models. In: Modeling and computational methods for kinetics equations. Model. Simul. Sci. Eng. Technol. Birkhäuser Boston, Boston, MA, 321-356 (2004)

21. Maugeri, A., Palagachev, D., Softova, L.: Elliptic and parabolic equations with discontinuous coefficients. Wiley, New York (2002)

22. Norris, J.R.: Brownian coagulation. Commun. Math. Sci. 2(Supplemental Issue) 93-101 (2004)

23. Pierre, M.: Global existence in reaction-diffusion systems with control of mass: a survey. Milan J. Math. 78(2), 41-455 (2010)

24. Rezakhanlou, F.: Moment bounds for the solutions of the Smoluchowski equation with coagulation and fragmentation. Proc. R. Soc. Edinb.: Sect. A Math. 140(5), 1041-1059 (2010)

25. Rezakhanlou, F.: Pointwise bounds for the solutions of the Smoluchowski equation with diffusion. Arch. Ration. Mech. Anal. 212(3), 1011-1035 (2014)

26. Smoluchowski, M.: Drei Vorträge über Diffusion, Brownsche Molekularbewegung und Koagulation von Kolloidteilchen. Physik. Zeitschr. 17, 557-599 (1916)

27. Smoluchowski, M.: Versuch einer mathematischen Theorie der Koagulationskinetik kolloider Lösungen. Zeitschrift f. physik. Chemie 92, 129-168 (1917)

28. White, W.H.: A global existence theorem for Smoluchowski's coagulation equations. Proc. Am. Math. Soc. 80, 273-276 (1980)

29. Wrzosek, D.: Existence of solutions for the discrete coagulation-fragmentation model with diffusion. Topol. Methods Nonlinear Anal. 9(2), 279-296 (1997)

30. Wrzosek, D.: Weak solutions to the Cauchy problem for the diffusive discrete coagulationfragmentation system. J. Math. Anal. Appl. 289(2), 405-418 (2004)

31. Wrzosek, D.: Mass-conserving solutions to the discrete coagulation fragmentation model with diffusion. Nonlinear Anal. 49, 297-314 (2002) 